\title{
Paraffin oil based soft-template approach to fabricate reusable porous PDMS sponge for effective oil/water separation
}

Leicheng Zhang, Yifang Zhang, Peng Chen, Wei Du, Xiaojun Feng, Bi-Feng Liu*

The Key Laboratory for Biomedical Photonics of MOE at Wuhan National Laboratory for Optoelectronics-Hubei Bioinformatics \& Molecular Imaging Key Laboratory, Systems Biology Theme, Department of Biomedical Engineering, College of Life Science and Technology, Huazhong University of Science and Technology, Wuhan 430074, China

*Corresponding author

Add: Department of Biomedical Engineering

College of Life Science and Technology

Huazhong University of Science and Technology, Wuhan 430074, China

Email: bfliu@mail.hust.edu.cn

Tel: $\quad+86-27-87792203$

Fax: $\quad+86-27-8779217$ 


\section{List of Figure as Supporting Information}

Results and discussion of magnetic PDMS sponge Page S-3

Table S1. Preparation conditions and the properties of the PDMS sponges .....................Page S-4

Table S2. Preparation conditions at $m_{\mathrm{PDMS}} / m_{\text {paraffin }}=2: 3$ and the properties of PDMS sponges ......Page S-4 Figure S1. Photograph of diluted PDMS solution, PDMS-water emulsion and PDMS sponge ......Page S-5

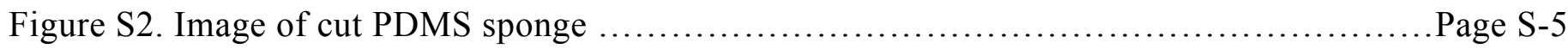

Figure S3. Micrograph of patterned PDMS sponge surface $\ldots \ldots \ldots \ldots \ldots \ldots \ldots \ldots \ldots \ldots \ldots \ldots \ldots \ldots$. Page S-6

Figure S4. Photograph of the PDMS-Ni sponge (water contact angle and magnetic force) ......Page S-6 Movie S1. Removal of hexane on the water surface with PDMS sponge ......................Page S-7 Movie S2. Removal of chloroform under the water with PDMS sponge .....................Page S-7 


\section{Results and discussion of magnetic PDMS sponge}

Preparation of the PDMS-Ni sponge. Magnetic porous materials have received great attentions because they could be used for environmental pollution treatment by simple magnetic interaction. However, the conventional methods for fabricating magnetic porous material are time-consuming and complicated. Here, we proposed a simple approach for synthesis of magnetic porous material based on PDMS and Ni nanoparticles via emulsion technique. The PDMS-Ni sponges were prepared according to the section 2.2 protocol, only with an addition of $2 \mathrm{~g}$ Ni powder during emulsification process $\left(m_{\mathrm{Ni}} / m_{\mathrm{PDMS}} / m_{\text {paraffin }} / m_{\text {water }}=2\right.$ : 5 : 7.5 : 35). After emulsifying, the PDMS-Ni-water emulsion was uniform and stable during the polymerization process. The as-obtained emulsions were heated at $65{ }^{\circ} \mathrm{C}$ for $2 \mathrm{~h}$ to accomplish polymerization. Thus, the emulsion finally transformed into PDMS-Ni nanocomposite. After repeated washing with ethanol to remove paraffin oil and un- entrapped Ni nanoparticles, a flexible and porous magnetic sponge was obtained. Figure S4b described the wettability of PDMS-Ni sponge. A $5 \mu \mathrm{L}$ water drop on the PDMS-Ni sponge surface were spherical in shape, but an oil drop with the same volume was quickly absorbed into the sponge, which indicated that the obtained magnetic sponge possesses efficient oil/water mixture separation capability. The PDMS-Ni sponge was endowed magnetic performance (Figure S4c, Supporting Informatiom). Therefore, as the simple fabrication, great magnetic performance, and excellent hydrophobic/oleophilic, the PDMS-Ni sponges would be a promising candidate for oil spill reclamation. 
Table S1. Preparation conditions and the properties of the PDMS sponges

\begin{tabular}{lcccccc}
\hline samples & PDMS $(\mathrm{g})$ & $\begin{array}{c}\text { Paraffin } \\
\text { oil }(\mathrm{g})\end{array}$ & $\begin{array}{c}\text { Water }^{\mathrm{b}} \\
(\mathrm{g})\end{array}$ & $\begin{array}{c}\text { Porosity } \\
(\%)\end{array}$ & $\begin{array}{c}\text { Ultimate stress } \\
(\mathrm{MPa})\end{array}$ & $\begin{array}{c}\mathrm{CA}_{\text {water }} \\
\left({ }^{\circ}\right)\end{array}$ \\
\hline PDMS 1\# & 5 & 0 & $11 \pm 1.5$ & $55 \pm 0.8$ & $2.69 \pm 0.321$ & $116.4 \pm 1.8$ \\
PDMS 2\# & 5 & 1 & $20 \pm 1.2$ & $69.3 \pm 0.7$ & $2.19 \pm 0.242$ & $121.3 \pm 1.7$ \\
PDMS 3\# & 5 & 2 & $26 \pm 2.2$ & $73.6 \pm 1.2$ & $2.04 \pm 0.315$ & $122.6 \pm 2.6$ \\
PDMS 4\# & 5 & 3 & $30 \pm 2.5$ & $77.8 \pm 2.3$ & $1.71 \pm 0.097$ & $122.7 \pm 1.2$ \\
PDMS 5\# & 5 & 5 & $34 \pm 1.5$ & $80.3 \pm 1.6$ & $0.97 \pm 0.029$ & $123.8 \pm 1.7$ \\
PDMS 6\# & 5 & 7.5 & $43^{\mathrm{b}} \pm 2.4$ & $82.2 \pm 2.2$ & $0.14 \pm 0.005$ & $125.5 \pm 2.4$ \\
PDMS 7\# & 5 & 8.5 & $52^{\mathrm{b}} \pm 3.2$ & $39.5 \pm 1.9$ & - & - \\
PDMS 8\# & 5 & 11 & $60^{\mathrm{b}} \pm 3.5$ & $20.7 \pm 0.5$ & - & - \\
\hline
\end{tabular}

${ }^{\mathrm{b}}$ The amount of emulsion water was at the saturated emulsion (PDMS 6\#, 7\#, 8\#, were less $6 \mathrm{~g}$ than the saturated emulsion water).

-The bar indicates that the sample was not suitable to measure the stress-strain curve and $\mathrm{CA}_{\text {water }}$.

Table S2. Preparation conditions at $m_{\mathrm{PDMS}} / m_{\text {paraffin }}=2: 3$ and the properties of PDMS sponges

\begin{tabular}{ccccccc}
\hline samples & PDMS (g) & $\begin{array}{c}\text { Paraffin } \\
\text { oil }(\mathrm{g})\end{array}$ & $\begin{array}{c}\text { Water } \\
(\mathrm{g})\end{array}$ & $\begin{array}{c}\text { Porosity } \\
(\%)\end{array}$ & $\begin{array}{c}\text { Ultimate stress } \\
(\mathrm{MPa})\end{array}$ & $\begin{array}{c}\mathrm{CA}_{\text {water }} \\
\left({ }^{\circ}\right)\end{array}$ \\
\hline PDMS 9\# & 5 & 7.5 & 27 & $82.1 \pm 1.6$ & $0.091 \pm 0.007$ & $124.3 \pm 5.3$ \\
PDMS 10\# & 5 & 7.5 & 31 & $84.5 \pm 1.2$ & $0.084 \pm 0.005$ & $128.9 \pm 1.8$ \\
PDMS 11\# & 5 & 7.5 & 35 & $85.6 \pm 1.6$ & $0.069 \pm 0.009$ & $141.9 \pm 2.5$ \\
PDMS 12\# & 5 & 7.5 & 39 & $83.2 \pm 1.3$ & $0.086 \pm 0.012$ & $124.7 \pm 5.5$ \\
PDMS 6\# & 5 & 7.5 & 43 & $82.2 \pm 2.3$ & $0.144 \pm 0.005$ & $125.5 \pm 4.1$ \\
PDMS 13\# & 5 & 7.5 & 46.9 & $8.80 \pm 0.6$ & - & - \\
\hline
\end{tabular}

-The bar indicates that the sample was not suitable to measure the stress-strain curve and $\mathrm{CA}_{\mathrm{water}}$. 


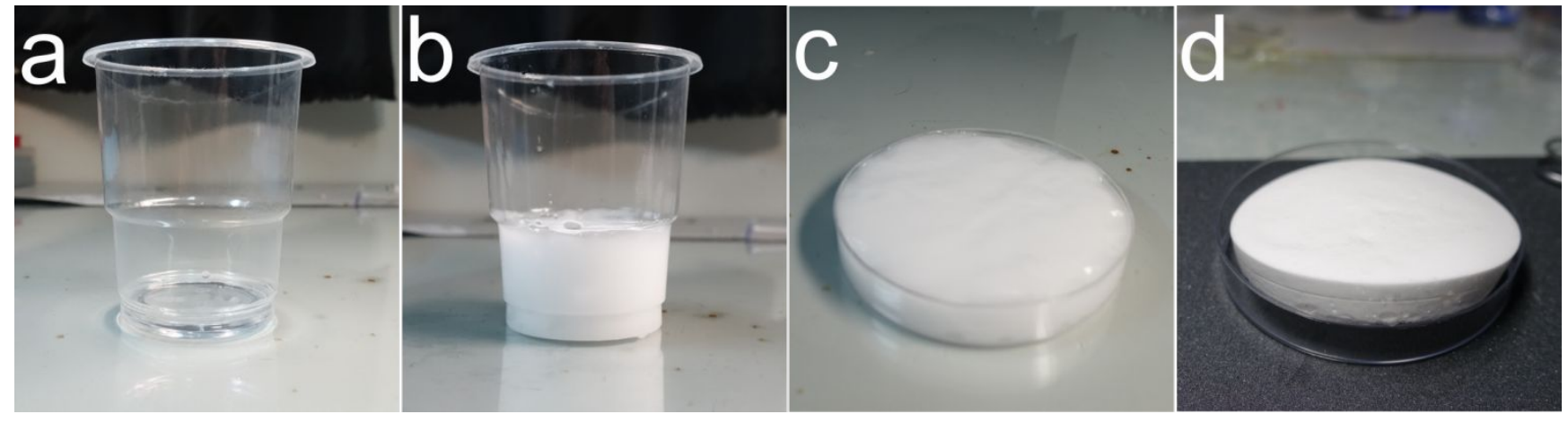

Figure S1. (a) Photograph of the PDMS pre-polymer diluted with paraffin oil. (b) Photograph of the freshly prepared PDMS-water emulsions. (c) PDMS-water emulsion poured into the petri dish. (d) Image of the synthesized PDMS sponge.
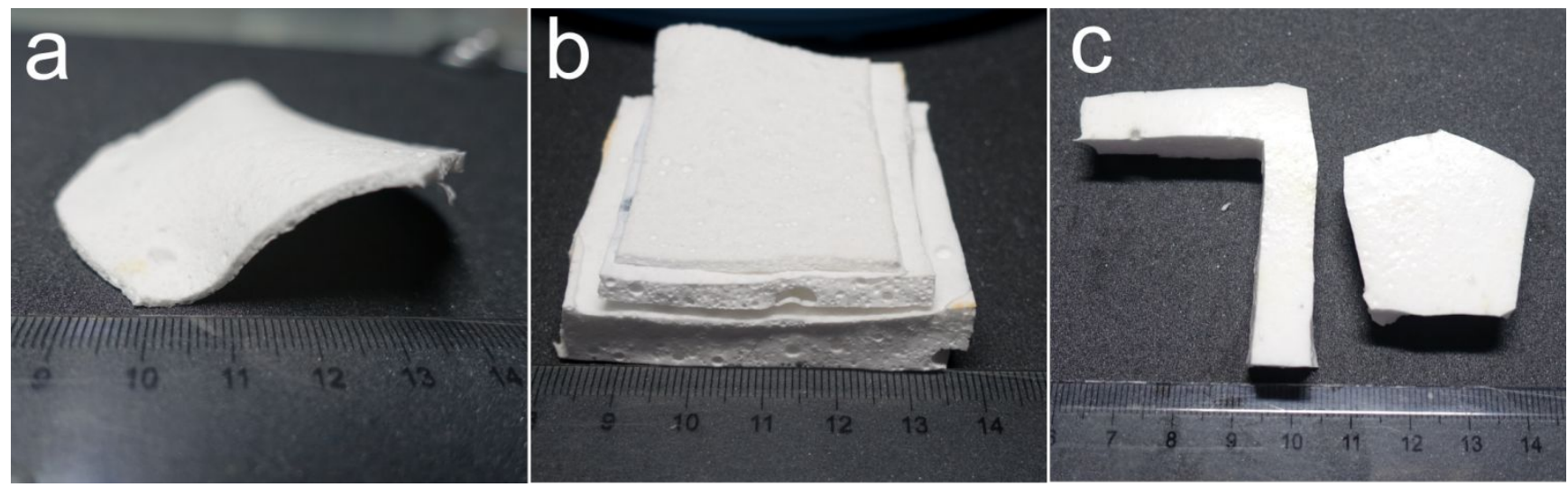

Figure S2. (a-c) Image of cut PDMS sponge with different thickness. 


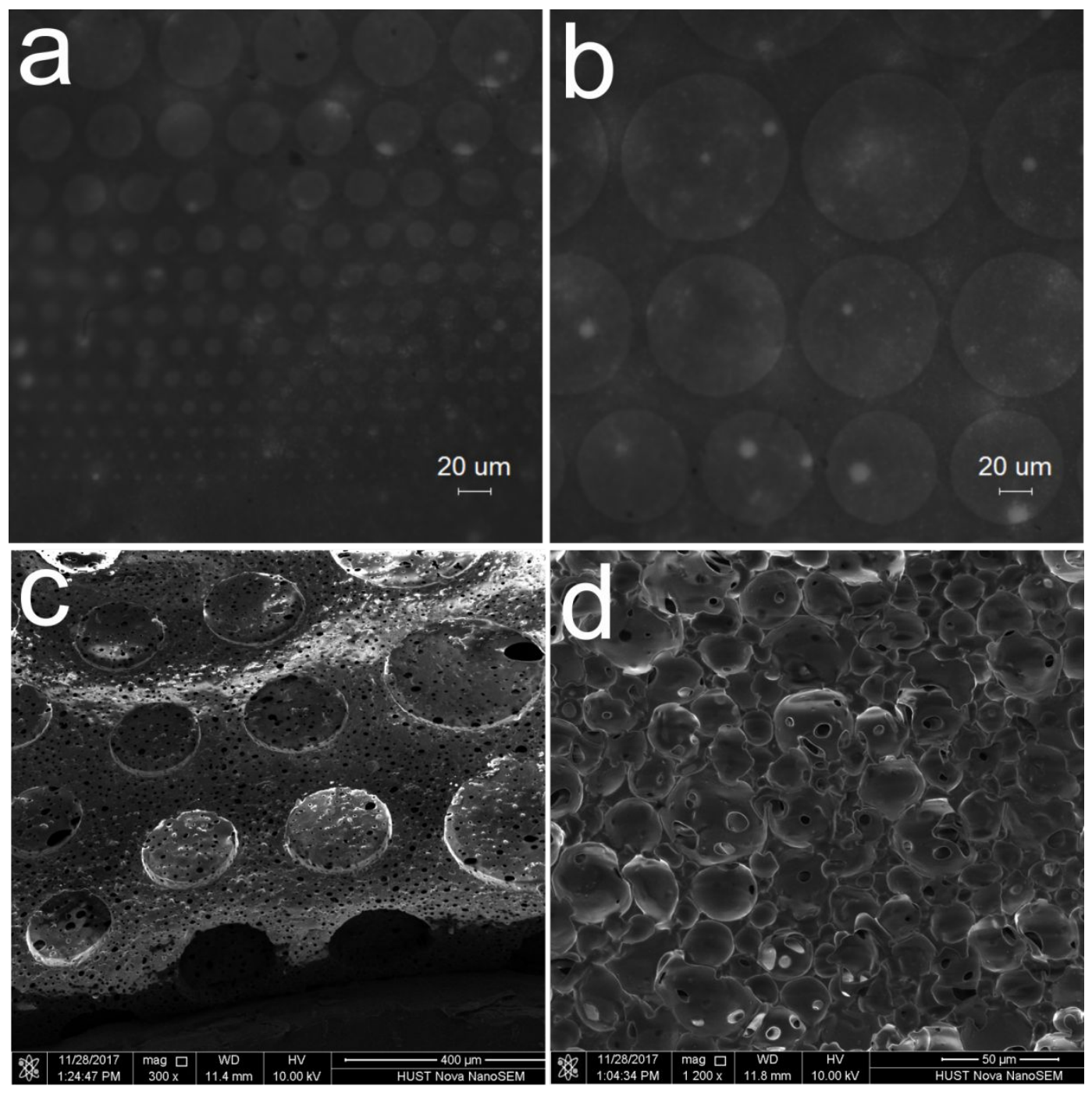

Figure S3. (a-b) Micrograph of microwells on the PDMS sponge surface. SEM image of the microwells on the surface of the PDMS sponge (c) and cross-sectional section of the PDMS sponge (d).

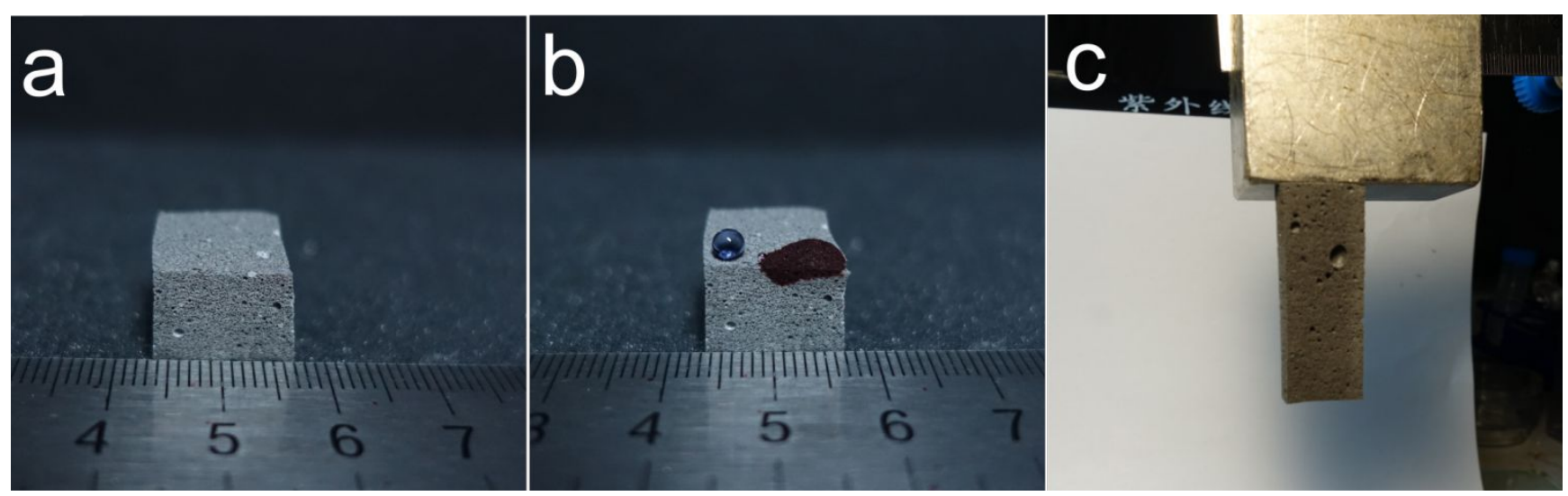

Figure S4. (a) Photograph of the PDMS-Ni sponge. (b) High hydrophobicity and strong oleophilicity of the surface. (c) Photograph of the PDMS-Ni sponge anchored to a magnet. Water and Oil were dyed with methylene blue and Oil Red O, respectively. 
Movie S1 Removal of hexane on the water surface with PDMS 11\# sponge. Hexane was colored with Oil Red O.

Movie S2 Removal of chloroform under the water with PDMS 11\# sponge. Chloroform was colored with Oil Red O. 